\title{
A Skew-Slit Collimator for Small-Animal SPECT
}

\author{
Gengsheng L. Zeng \\ Department of Radiology, Utah Center for Advanced Imaging Research (UCAIR), University of Utah, Salt Lake City, Utah
}

The main objective of this work was the development of a skewslit collimator for small-animal SPECT to replace the state-ofthe-art multipinhole collimator. Methods: A pinhole forms a cone-beam imaging geometry. If the collimator rotates around the object in a circular orbit, the projection measurements acquired by the cone-beam imaging geometry are incomplete and not enough for artifact-free image reconstruction. The severity of the artifact is proportional to the cone angle of the pinhole in the direction of the axis of rotation. Multipinhole geometry can greatly reduce the data-insufficiency artifacts; however, image magnification is sacrificed. By transforming a pinhole into a pair of skewed slits, we are able to use a large cone angle in the transaxial direction to increase image magnification and a small cone angle in the direction of the axis of rotation to reduce data-insufficiency artifacts. This transformation is achieved by placing a vertical slit (i.e., the slit is parallel to the axis of rotation) close to the object and placing a horizontal slit farther out. Similar to the multipinhole collimator, we also propose a multiple-skew-slit collimator that has a single vertical slit and several horizontal slits. Results: Computer simulations were performed to verify the working principle of the skew-slit collimator. A prototype multiple-skew-slit collimator was fabricated, and phantom experiments were performed on a SPECT system. The smallest channels (of $0.75-\mathrm{mm}$ diameter) in the phantom were clearly separated in the reconstructed image. Conclusion: This study suggests a novel technology in smallanimal SPECT to replace the multipinhole collimator, resulting in significantly reduced image artifacts and increased transaxial resolution. A physical multiple-skew-slit system was built and tested. Compared with the multipinhole system, the multipleskew-slit system has a larger image magnification in the transaxial direction and thus has better image resolution.

Key Words: molecular imaging; SPECT; instrumentation; pinhole; slit collimator; small-animal SPECT

J Nucl Med Technol 2008; 36:207-212

DOI: $10.2967 /$ jnmt.108.055582

I n SPECT cameras, the spatial resolution depends mainly on the geometry of the collimation system. Theoretically, it should be possible to produce cameras yielding submillimetric spatial resolution using collimators with holes of small diameter. However, the low efficiency that should result from such a geometry would impose nonrealistic acquisition times

Received Jun. 30, 2008; revision accepted Sep. 23, 2008.

For correspondence contact: Gengsheng L. Zeng, Department of Radiology, Utah Center for Advanced Imaging Research (UCAIR), University of Utah, 729 Arapeen Dr., Salt Lake City, UT 84108.

E-mail: larry@ucair.med.utah.edu

COPYRIGHT @ 2008 by the Society of Nuclear Medicine, Inc. and require high radionuclide doses. High-resolution SPECT systems are useful in tracer development and preclinical research where new radiopharmaceuticals have to be tested and evaluated in small-animal studies. Recent developments in the use of pinhole SPECT for small-animal imaging have identified a technology that can be used to provide images with a spatial resolution on the order of $0.6 \mathrm{~mm}$ (1). In SPECT, the system detection sensitivity and spatial resolution are determined mainly by the collimator. Parallel-hole collimators are routinely used in SPECT scans. However, for small-object imaging, parallel-hole collimation is not geometrically efficient. As an alternative, convergent collimators such as conebeam or pinhole collimators can offer better geometric efficiency, with an improvement of spatial resolution and sensitivity. Given a specified spatial resolution, the cone-beam collimator has a higher sensitivity than the pinhole collimator for larger objects (e.g., object diameters larger than $5 \mathrm{~cm}$ ), whereas the pinhole collimator has a higher sensitivity than the cone-beam collimator for smaller objects (e.g., object diameter smaller than $3 \mathrm{~cm}$ ) (2). Therefore, the pinhole collimator has been chosen for small-animal SPECT. Another advantage of the pinhole collimator is that it is easy to make a multipinhole collimator to provide increased sensitivity. In small-animal SPECT, the limitations currently relate largely to sensitivity. Several novel collimation techniques are being explored that can simultaneously provide high resolution and relatively high sensitivity by using multiple pinholes or coded aperture methods. Schramm et al. used a multipinhole $\left(60^{\circ}\right.$ cone angle, $1.5-\mathrm{mm}$ hole diameter) collimator and achieved a 2-mm image resolution with some multiplexing allowed on the detector (3). Beekman et al. later proposed a rotationless U-SPECT-I multipinhole system $\left(30^{\circ}\right.$ cone angle, 0.6-mm hole diameter; Molecular Imaging Laboratories) that reached 0.5 -mm image resolution with no multiplexing allowed (I). A SPECT device with sliding slit and parallel rake collimator, called Linoview (fanbeam geometry; Linoview Systems), was attempted by Walrand et al. recently, and submillimeter resolution was also obtained (4). The pinhole system has its drawbacks. One problem is the pinhole's cone-beam imaging geometry, which does not provide a complete dataset as the detector rotates around the object. A complete dataset is required for an artifact-free quantitative reconstruction. A larger cone angle (i.e., the pinhole acceptance angle) gives more severe artifacts. One remedy is to use a nonplanar scanning orbit, for example, a helical orbit or a circle-and-line orbit, that, however, complicates the imaging systems (5). The 
state-of-the-art multipinhole geometry can mitigate the datainsufficiency problem by using smaller cone angles. However, the smaller cone angle (i.e., smaller image magnification on the detector) may result in poor spatial resolution. The relation between the vertical cone angle and the data sufficiency requirement can be explained by Palamodov's condition ( 6 ), which can be roughly stated as follows: every plane that cuts the object must contain a measured straight line. When the vertical cone angle gets larger, more planes that cut the object do not contain a measured line. Our group has developed a skew-slit collimator that is an extension of the pinhole collimator (7-10). The skew-slit system is similar to a pinhole system, with the advantage of independent adjustment of cone angles in the axial and transaxial directions. Because the distortion and artifacts are caused by the large cone angle in the axial direction, we proposed to use a relatively small cone angle in the axial direction and a relatively large cone angle in the transaxial direction. A multiple-skew-slit geometry has high detection sensitivity, small axial magnification (i.e., less severe data-insufficiency artifacts), and large transaxial magnification (i.e., better image resolution). It is expected that the multiple-skew-slit system will have better transaxial resolution than that of existing multipinhole systems. The skew-slit collimator is also related to the slit-slat collimator (11-14). In a slit-slat system, the slit is equivalent to the vertical slit in our skew-slit system, and the slat collimator provides a set of parallel 1-dimensional fanbeam subsystems. The slit-slat system has a large image magnification in the transaxial direction and no magnification in the axial direction.

\section{MATERIALS AND METHODS}

\section{Concept of Skew-Slit Collimator}

In a conventional pinhole imaging system, the cone angle (also known as pinhole acceptance angle) is isotropic, and its "footprint" is circular on the detector. In a skew-slit system, the cone angle is determined by 2 orthogonally oriented slits, as shown in Figure 1. The skew-slit system can be thought of as a generalized pinhole system. The vertical slit, which is parallel to the axis of rotation, is positioned close to the object and controls image magnification in the horizontal (i.e., the transaxial) direction. The magnification factor is the ratio of the distance between the slit and the detector to the distance between the slit and the object. The horizontal slit, which is orthogonal to the axis of rotation, is positioned between the vertical slit and the detector. The horizontal slit controls the image magnification factor in the vertical (i.e., the axial) direction. The proposed setup makes the image magnification in the axial direction smaller than the magnification in the transaxial direction. The skewslit concept is our original idea. As a special case, when there is no gap between the 2 slits, the 2 orthogonal focal lines become a focal point and the skew-slit system degenerates to a pinhole system.

In a pinhole system, a large cone angle gives a large image magnification, which results in improved detection sensitivity and better spatial resolution. On the other hand, a large cone angle causes a severe data-insufficiency problem, which results in distortion and artifacts in the reconstructed 3D image (Fig. 2). The skew-slit system allows the magnification factors in the axial and transaxial directions to be changed independently. Thus we can use a larger cone angle in the transaxial direction for a large image magnification and large detection sensitivity and a smaller cone angle in the axial direction for artifact and distortion control. The multipinhole collimator is able to significantly reduce cone-beam artifacts and to increase the detection sensitivity by tiling the detector with multiple cone-beam images. To avoid multiplexing as much as possible, the cone-beam image magnification in the multipinhole system is smaller than that in the single-pinhole system. The lesser image magnification could result in poorer spatial resolution. In the multiple-skew-slit collimator, there is a single vertical slit and several horizontal slits. Compared with the multipinhole system, the multiple-skew-slit system has a greater image magnification in the transaxial direction and thus has better image resolution. The multiple-skew-slit system has 3 distinct advantages over the state-of-the-art multipinhole system: First, the skew-slit system has a larger transaxial magnification factor and thus has better transaxial resolution. Second, it is much easier to design a multiple-skew-slit system with maximum detector area usage and minimum multiplexing. Third, the multiple projection images on the detector are easily separated, making possible the development of analytic reconstruction algorithms.

The disadvantage of the skew-slit collimator is the increased difficulty of imaging geometry parameter estimation, because more parameters such as slit tilt angles are used in the skew-slit collimator than in the pinhole collimator (15).

\section{Fabrication of a Prototype Multiple-Skew-Slit Collimator}

We have recently constructed a prototype of the multiple-skewslit collimator and performed phantom experiments (8). Figure 3
FIGURE 1. If spheric object is imaged, conventional pinhole system shows circular image (A), whereas skew-slit system shows short-and-fat elliptic image (B). Multipinhole system produces multiple almost-circular images (C). Multipleskew-slit system consists of single vertical slit and multiple horizontal slits, producing multiple elliptic images (D).

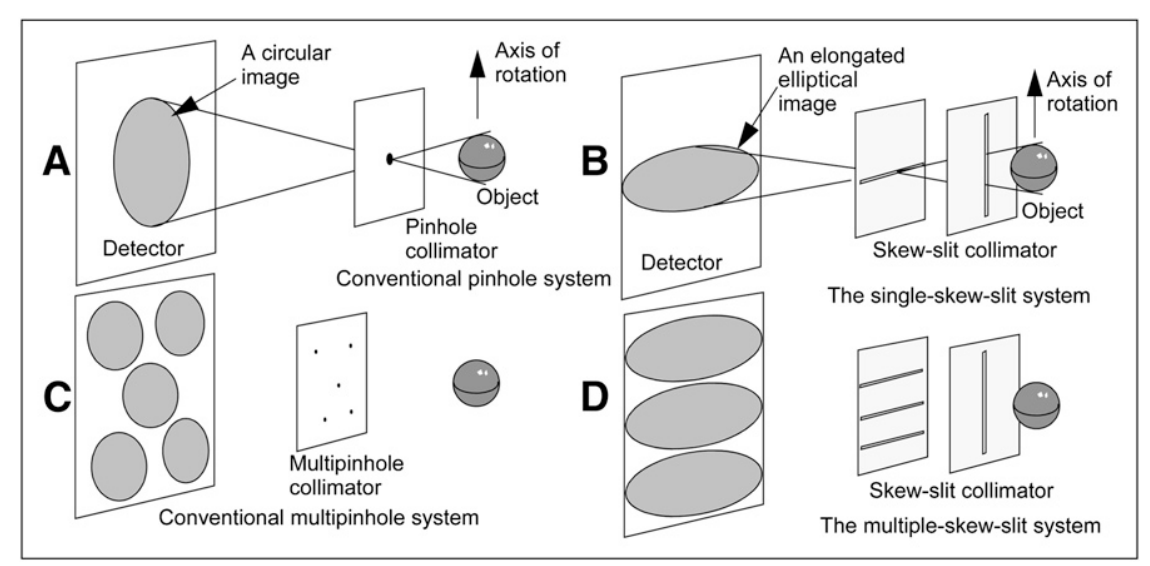




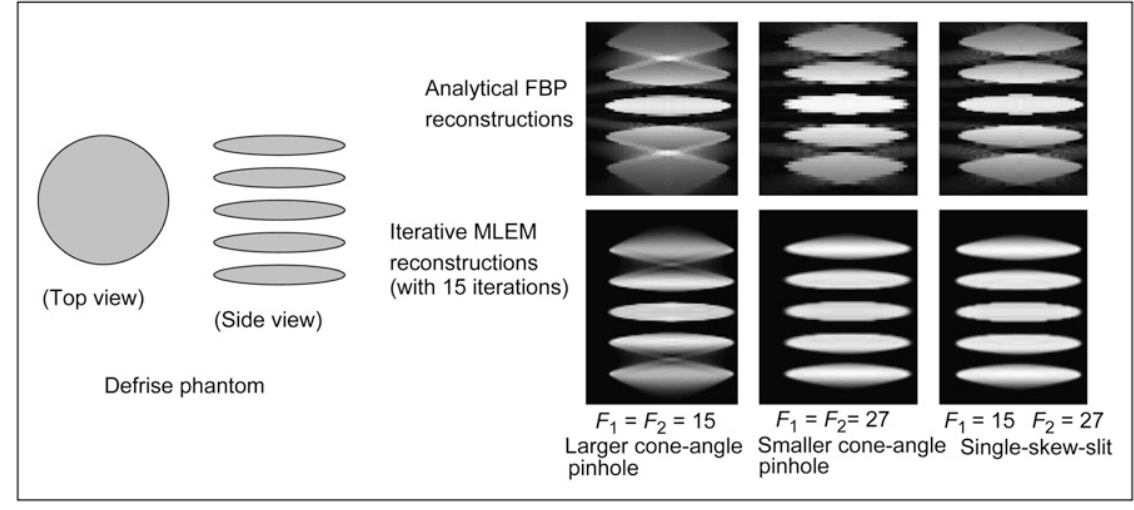

FIGURE 2. Defrise phantom and its reconstructions with analytic and iterative algorithms. Smaller axial cone angles give less severe cone-beam data-insufficiency artifacts. shows a cut-away drawing and a photograph of the prototype collimator. This collimator is designed for low-energy (e.g., 140 $\mathrm{keV}) \gamma$-ray imaging and has a lead housing. The slits are made of 4-mm-thick tungsten. The slit widths and positions are adjustable by screws. The default slit width is $0.5 \mathrm{~mm}$, and the slit acceptance angle is $90^{\circ}$. The default distance between the vertical slit and the horizontal slits is $9 \mathrm{~cm}$, and the fixed distance from the vertical slit to the detector surface is $30 \mathrm{~cm}$. One vertical and 3 horizontal slits are used. The collimator is designed to mount on an IRIX SPECT system (Philips).

\section{Computer Simulation 1: Artifact Study}

Computer simulations were performed to illustrate the effect of image artifacts as the result of collimator geometric parameters, which are labeled in Figure 4. When $F_{1}=F_{2}$ (where $F_{1}$ is vertical-slit focal length and $F_{2}$ is horizontal-slit focal length), the skew-slit collimator degenerates into a pinhole collimator. The projection data were analytically calculated and were free from attenuation, scattering, and noise. The data were generated on a $129 \times 129$ array with 120 angles uniformly distributed over $360^{\circ}$, using $D_{1}+F_{1}=D_{2}+F_{2}=100$ units (where $D_{1}$ and $D_{2}$ are the distances between the detector and the vertical and horizontal slits, respectively.). Here, the unit is the detector bin size. A single vertical slit and 5 horizontal slits were used. Three-dimensional images were reconstructed with an in-house filtered backprojection algorithm (7) and the well-known iterative maximum-likelihood expectation maximization algorithm (16).

\section{Computer Simulation 2: Resolution Comparison}

Another set of computer simulations was performed to compare multipinhole (with 5 pinholes) and multislit (with 3 horizontal slits) collimators in terms of image resolution. We considered an ideal collimation with a zero aperture. The resolution degradation is caused only by conversion to discrete variables on the $128 \times 128$ detector. $F_{1}$ was 17 units, $F_{2}$ was 39 units, and the pinhole focal length was 39 units. The unit was the detector pixel size. Both geometries had the same magnification in the transaxial direction, as shown in the projections in Figure 5. Both imaging geometries had about the same detection sensitivity. The phantom had a uniform spheric background and small hot lesions of different sizes. The lesion activity density was twice the background activity density. The projections were generated analytically without noise added. The images were reconstructed with 4 iterations of the ordered-subsets expectation maximization algorithm (17).

\section{Phantom Experiment}

An ultra-micro hot-spot phantom (Fig. 6A; Data Spectrum Corp.) filled with $296 \mathrm{MBq}$ of ${ }^{99 \mathrm{~m}} \mathrm{Tc}$ was used for the phantom study and was scanned on the IRIX SPECT system. The vertical slit width was set at $0.5 \mathrm{~mm}$, and the horizontal slit width was set at $1 \mathrm{~mm}$. In this Jaszczak phantom experiment, we cared more about the resolution in the transaxial direction than that in the axial direction. Thus, the width of the vertical slit was set smaller than the width of the horizontal slits. The adjacent gap between

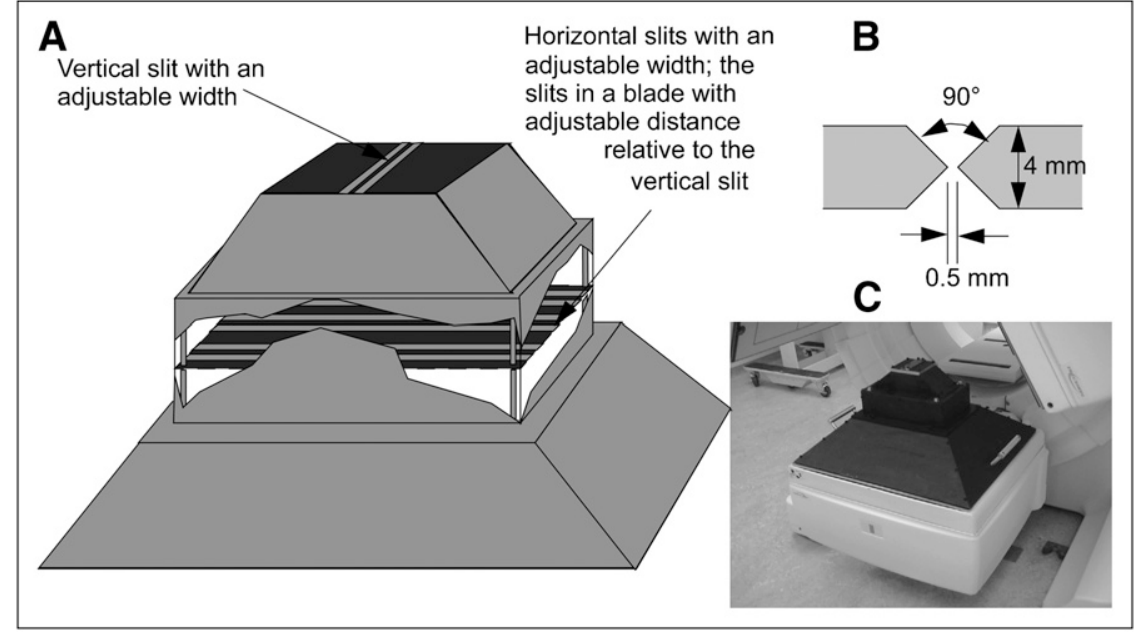

FIGURE 3. (A) Design of prototype skew-slit collimator. (B) Close-up view of slit cross section. (C) Photograph of prototype collimator mounted on IRIX SPECT scanner (Philips). 
FIGURE 4. Axial and transaxial amplification factors depend on slit locations $F_{1}$ and $F_{2}$.

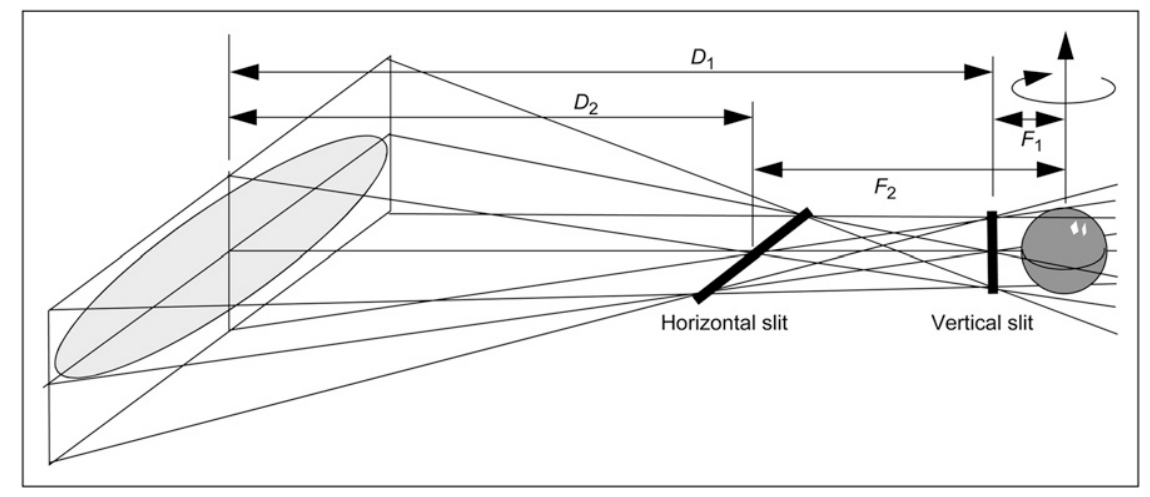

the 3 horizontal slits was $10 \mathrm{~mm}$ so that the projection data on the detector from these 3 horizontal slits did not overlap.

The phantom had 6 sections of hollow channels, with channel diameters of $2.4,2.0,1.7,1.35,1.0$, and $0.75 \mathrm{~mm}$. The outside diameter of the phantom was $3.5 \mathrm{~cm}$, and the outside height of the phantom was $5.5 \mathrm{~cm}$. The detector array had a size of $256 \times 256$, and the image array had a size of $256 \times 256 \times 256$. The pixel size in the detector array was $2.3 \mathrm{~mm}$. The focal lengths were $F_{1}=3.5$ $\mathrm{cm}, F_{2}=16 \mathrm{~cm}, D_{1}=30.5 \mathrm{~cm}$, and $D_{2}=18 \mathrm{~cm}$.

The phantom was scanned at 180 views over $360^{\circ}$, at $28 \mathrm{~s}$ per view using the step-and-shoot mode. The ordered-subsets expectation maximization algorithm (17) was used to reconstruct the image (10 iterations).

\section{RESULTS}

\section{Computer Simulation 1: Artifact Study}

Figure 2 shows that a larger axial cone angle gives less severe cone-beam data-insufficiency artifacts. From Figure 2, one also observes that if the pinhole cone angle is the same as the vertical cone angle of the skew slit, they suffer the same amount of data-insufficiency artifacts. The image artifacts are also dependent on the reconstruction algorithm. Iterative algorithms in general give fewer artifacts than analytic algorithms, especially when projection data are incomplete.

\section{Computer Simulation 2: Resolution Comparison}

When both multipinhole and multiple-skew-slit geometries had the same magnification in the transaxial direction and both imaging geometries had about the same detection sensitivity, it is clear from the profile comparison in Figure 5 that the multiple-slit system gave better spatial resolution in the transaxial plane.

\section{Phantom Experiment}

The phantom experiment results are shown in Figure 6. Figure 6B shows a single-detector view, in which 3 projection images are captured with no overlaps. The projection images appear short and fat. This means that the collimator has a much larger magnification factor in the transaxial direction than in the axial direction. The channels with the smallest diameter $(0.75 \mathrm{~mm})$ in the phantom were clearly separated in the reconstruction shown in Figure 6C, implying that the image resolution is approximately $0.5 \mathrm{~mm}$.

\section{DISCUSSION}

The skew-slit collimator is flexible for imaging small objects and small animals. The vertical slit controls the transaxial resolution, and the horizontal slits control the
FIGURE 5. Comparison study between multipinhole system and multiple-skewslit system. (A) One-projection view of 5-pinhole system. (B) One-projection view of 3-horizontal-slit system. (C) Central slice of reconstruction from pinhole data. (D) Central slice of reconstruction from skew-slit data. (E) Profiles across reconstructions through small lesions (solid line, skew-slit image; dotted line, pinhole image).

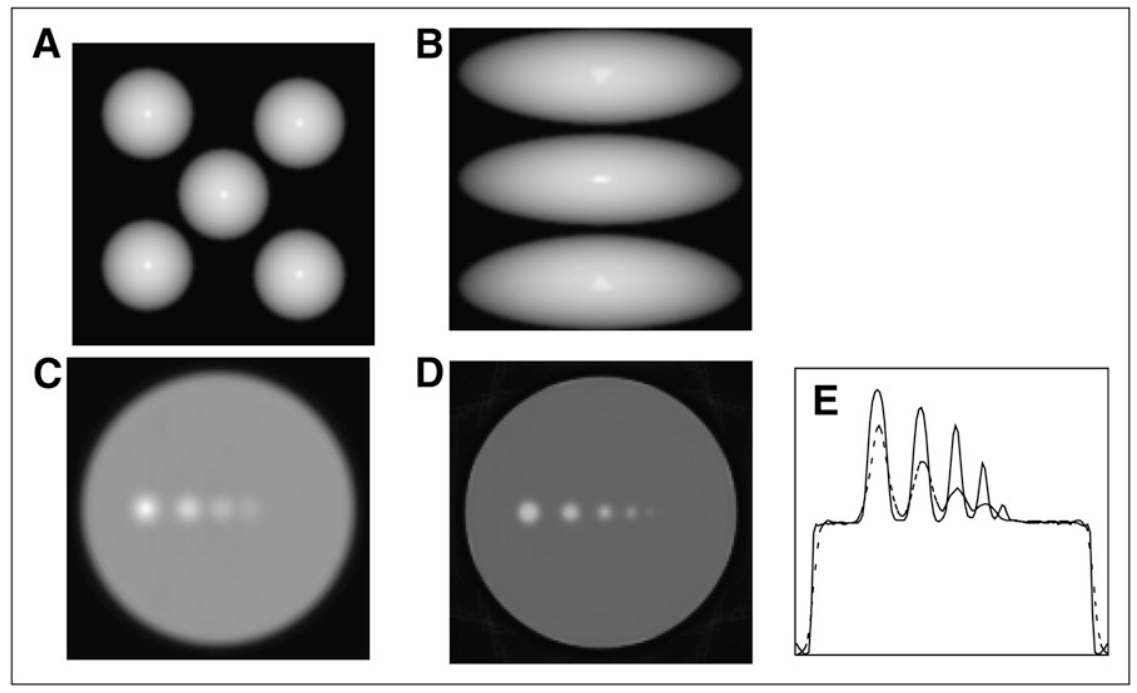



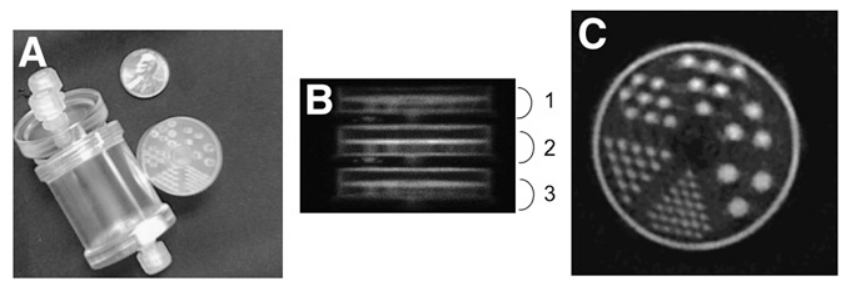

FIGURE 6. (A) Photograph of phantom and penny. (B) Projection view, where nonoverlapping images from 3 horizontal slits are labeled 1, 2, and 3. (C) Ordered-subsets expectation maximization reconstruction, where smallest structure can be resolved.

axial resolution. In a skew-slit imaging system, the image magnification factor in the axial direction is greater than that in the transaxial direction. This system intrinsically has better resolution in the transaxial direction than in the axial direction. If a particular study requires isotropic reconstructed image resolution, the slit width $d_{H}$ of the horizontal slit will be set smaller than the slit width $d_{V}$ of the vertical slit. The ratio $d_{H} / d_{V}$ should be set as the ratio of the image magnification factor for the vertical slit over the image magnification factor for the horizontal slits; that is,

$$
\frac{d_{H}}{d v}=\frac{D_{1}+F_{1}}{F_{1}} / \frac{D_{2}+F_{2}}{F_{2}} .
$$

The above relation is an approximation because the images due to different slits are not identical and some extra axial sampling information is obtained by using multiple horizontal slits. Another aspect is that the image system is rotational and the rotational blurring is decoded via the image reconstruction algorithm. In practice this decoding is never perfect, resulting in poorer resolution in the transaxial direction than in the axial direction.

\section{CONCLUSION}

Pinhole imaging has a significant role in small-animal SPECT. Thus, it is important to advance the technology to the next generation of collimation so that reconstruction artifacts and distortion can be significantly reduced and spatial resolution can be increased. Using a multiple-skewslit collimator is a new concept in this direction. To significantly reduce data-insufficiency artifacts, we require that the imaging cone angle in the axial direction be small. To efficiently use the detector surface area and reach a high detection sensitivity, we require that the projection data use up the detector surface as much as possible. Thus, we use a large imaging cone angle in the transverse direction and multiple horizontal slits.

To obtain projection data that contain maximal tomographic information, we require that the projection data from different horizontal slits overlap as little as possible. Thus, we use multiple horizontal slits separated by an optimal distance. In order to completely remove the datainsufficiency artifacts, a nonplanar gantry trajectory (e.g., a spiral trajectory) must be used. However, we believe that for small-animal SPECT studies, the multiple-skew-slit imaging geometry can reduce the data-insufficiency artifacts to a level such that the errors are insignificant and not noticeable. In this way, a complicated scanning geometry can be avoided and cost can be reduced. Multiple-skew-slit imaging may have different spatial resolutions in the axial and transaxial directions.

When multiple horizontal slits are used, the vertical resolution will be improved because the "duplicated" measurements from different slits do not have exactly the same sampling points and this difference gives an equivalent finer sampling in the axial direction. When we use multiple slits, multiple images are formed on the detector. It is important to realize that these multiple images are not identical copies of each other, because they are sampled at different locations. The result is that the effective sampling in the axial direction is finer than the actual sampling interval in the axial direction on the detector. Hence, the spatial resolution of a multiplehorizontal-slit system is better than that of a single-horizontalslit system. This effect has been confirmed by resolution measurements in computer simulations with iterative MLEM reconstructed images.

This paper focuses on the proof of the concept of a skew-slit collimator. The paper is by no means a rigorous evaluation of the multiple-skew-slit system. Attenuation, scattering, system blurring, and noise are not considered in this initial study and will be left for future development. Our future work will also include careful evaluation of the multipleskew-slit system and comparison with the state-of-the-art multipinhole system.

\section{ACKNOWLEDGMENTS}

This work was partially supported by NIH 5R21 EB006830 and by the Ben B. and Iris M. Margolis Foundation and the Benning Society. I thank James Wright and Jacob Piatt for assistance in collimator design and data acquisition. I also thank Dr. Roy Rowley for his assistance in English editing.

\section{REFERENCES}

1. Beekman FJ, van der Have F, Vastenhouw B, et al. U-SPECT-I: a novel system for submillimeter-resolution tomography with radiolabeled molecules in mice. J Nucl Med. 2005;46:1194-1200.

2. Zeng GL. Pinhole SPECT vs. cone-beam SPECT. In: Zhang D, ed. Medical Biometrics Lecture Notes in Computer Science 4901. Berlin, Germany: SpringerVerlag; 2007:240-247.

3. Schramm NU, Ebel G, England U, Schurrat T, Behe M, Behr TM. Highresolution SPECT using multi-pinhole collimation. IEEE Trans Nucl Sci. 2003;50:315-320.

4. Walrand S, Jamar F, de Jong M, Pauwels S. Evaluation of novel whole-body high-resolution rodent SPECT (Linoview) based on direct acquisition of linogram projections. J Nucl Med. 2005;46:1872-1880.

5. Metzler SD, Greer KL, Jaszczak RJ. Helical pinhole SPECT for small-animal imaging: a method for addressing sampling completeness. IEEE Trans Nucl Sci. 2003;50:1575-1583.

6. Palamodov VP. Inversion formulas for the three-dimensional ray transform. Mathematical Methods in Tomography, Lecture Notes in Mathematics 1498. In: Herman GT, Louis AK, Natterer F, eds. Berlin, Germany: Springer; 1991:53-56. 
7. Huang Q, Zeng GL. An analytical algorithm for skew-slit imaging geometry with non-uniform attenuation correction. Med Phys. 2006;33:997-1004.

8. Zeng GL, Piatt JA. First phantom experiment of skew-slit SPECT [abstract]. J Nucl Med. 2007;48(suppl 2):93P.

9. Tang Q, Zeng GL, Huang Q. An analytical algorithm for skew-slit collimator SPECT with uniform attenuation correction. Phys Med Biol. 2006;51:6199-6211.

10. Zeng GL, inventor; University of Utah, assignee. Skew slit collimator and method of use thereof. U.S. patent 7,388,207, June 17, 2008

11. Accorsi R, Novak JR, Ayan AS, Metzler SD. Derivation and validation of a sensitivity formula for slit-slat collimation. IEEE Trans Med Imaging. 2008;27:709-722.

12. Novak JR, Ayan AS, Accorsi R, Metzler SD. Verification of the sensitivity and resolution dependence on the incidence angle for slit-slat collimation. Phys Med Biol. 2008;53:953-966.
13. Metzler SD, Accorsi R, Novak JR, Ayan AS, Jaszczak RJ. On-axis sensitivity and resolution of a slit-slat collimator. J Nucl Med. 2006;47:18841890.

14. Kamali-Asl A, Sarkar S, Shahriari M, Agha-Hosseini H. Slit slat collimator optimization with respect to MTF. Appl Radiat Isot. 2005;62:461-468.

15. Piatt JA, Zeng GL. Estimation of skew-slit SPECT acquisition geometry using a single point source. In: Proceedings of the 9th International Meeting on Fully 3D Image Reconstruction in Radiology and Nuclear Medicine, Lindau, Germany, July 9-13, 2007;2007:281-284.

16. Lange K, Carson R. EM reconstruction algorithms for emission and transmission tomography. J Comput Assist Tomogr. 1984;8:306-316.

17. Hudson HM, Larki RS. Accelerated image reconstruction using ordered subsets of projection data. IEEE Trans Med Imaging. 1994;13:601-609. 